
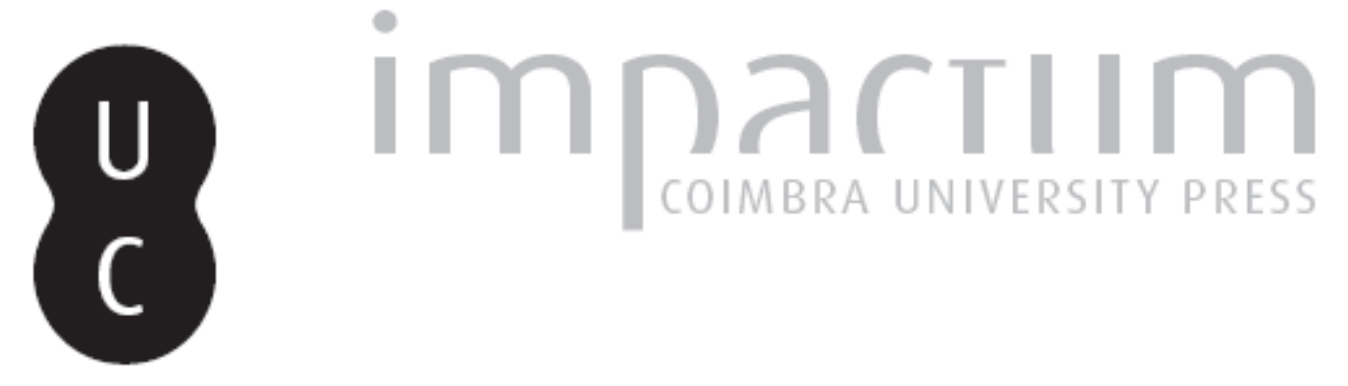

\title{
La mística plotiniana: experiencia, doctrina e interpretación
}

Autor(es): $\quad$ Martino, Gabriel

Publicado por: Annablume Clássica

URL persistente:

URI:http://hdl.handle.net/10316.2/24478

DOI:

DOI:http://dx.doi.org/10.14195/1984-249X_5_6

Accessed : $\quad$ 26-Apr-2023 11:07:04

A navegação consulta e descarregamento dos títulos inseridos nas Bibliotecas Digitais UC Digitalis, UC Pombalina e UC Impactum, pressupõem a aceitação plena e sem reservas dos Termos e Condições de Uso destas Bibliotecas Digitais, disponíveis em https://digitalis.uc.pt/pt-pt/termos.

Conforme exposto nos referidos Termos e Condições de Uso, o descarregamento de títulos de acesso restrito requer uma licença válida de autorização devendo o utilizador aceder ao(s) documento(s) a partir de um endereço de IP da instituição detentora da supramencionada licença.

Ao utilizador é apenas permitido o descarregamento para uso pessoal, pelo que o emprego do(s) título(s) descarregado(s) para outro fim, designadamente comercial, carece de autorização do respetivo autor ou editor da obra.

Na medida em que todas as obras da UC Digitalis se encontram protegidas pelo Código do Direito de Autor e Direitos Conexos e demais legislação aplicável, toda a cópia, parcial ou total, deste documento, nos casos em que é legalmente admitida, deverá conter ou fazer-se acompanhar por este aviso. 

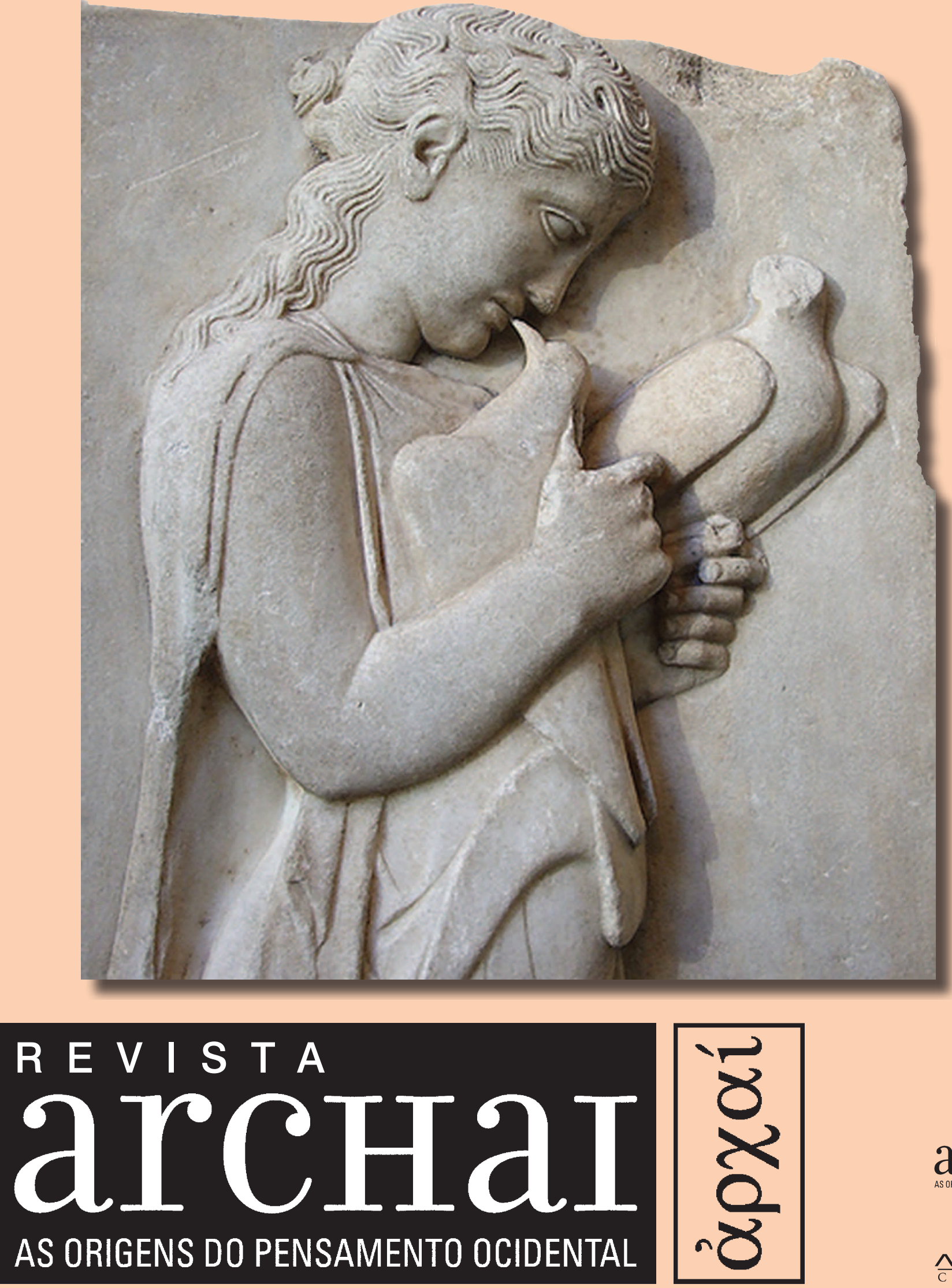


\section{LA MÍSTICA PLOTINIANA: EXPERIENCIA, DOCTRINA E INTERPRETACIÓN}

RESUMEN: Si bien el término "mística" no es propio de Plotino, la tradición interpretativa lo ha utilizado para caracterizar un aspecto de su filosofía. La mística plotiniana, pues, incluye aquellos pasajes de su obra en los que presenta o bien descripciones de diferentes experiencias trascendentes o bien su interpretación de estas. Sobre estos dos elementos, creemos, Plotino construye una doctrina coherente sobre la relación del hombre con los grados superiores de la realidad que es, a su vez, dependiente de su concepción metafisica.

En el presente trabajo nos proponemos brindar una caracterización ajustada de la doctrina mística plotiniana para lo cual será necesario analizar, asimismo, las particularidades de los diversos discursos místicos presentes en las Enéadas y brindar una sintesis de las principales líneas interpretativas que los estudiosos han establecido respecto de este tema.

PALABRAS CLAVE: Plotino - Experiencia mística - Neoplatonismo

ABSTRACT: Though "mysticism" in not a Plotinian idiom, it has been frequently used by scholars to identify an aspect of his philosophy. Thus, Plotinus' mysticism comprises those passages of the Enneads in which he describes different transcendent experiences or presents his interpretation of these. Upon these two elements, we believe, Plotinus structures a coherent doctrine about the relation between man and the higher degrees of reality closely dependant, as well, on his metaphysical theory.

In the present work we intend to provide an accurate account of Plotinus' mystical doctrine for which we will also need to analyse the singularities of the different mystical discourses present in the Enneads and outline and discuss the main streams of interpretation that scholars have suggested for this subject.

KEY WORDS: Plotinus - Mystical experience - Neoplatonism
Universidad de Buenos Aires - CONICET

\section{Gabriel Martino ${ }^{1}$}

La filosofía de Plotino, si bien es presentada como una exégesis de la filosofía platónica, tiene sus caracteres distintivos y particulares que hacen posible que Plotino haya sido considerado el fundador de una nueva corriente de pensamiento que lleva el discutido nombre de Neoplatonismo.

Uno de los caracteres propios de la filosofía plotiniana es su aspecto místico, tema que posee un desarrollo amplio y detallado aunque no falto de complejidad e, incluso, de ambigüedades. La complejidad y la ambigüedad, podría decirse, se deben a la conjunción de dos factores aparentemente en conflicto: el carácter inefable del objeto de estudio de la mística y el medio de expresión de esta, es decir, el discurso. A pesar de estas dificultades, consideramos que es una tarea valiosa intentar desentrañar los principios filosóficos sobre los que Plotino construye su doctrina mística.

En el presente trabajo nos proponemos, en primer lugar, exponer los caracteres generales de los discursos presentes en las Enéadas sobre las experiencias místicas $y$, en segundo lugar, brindar una síntesis de las principales líneas interpretativas que los estudiosos han establecido respecto de este tema. Aunque se han dedicado numerosos trabajos a esta temática en el siglo pasado, durante los últimos años la discusión en torno a la mística plotiniana 
pareciera haber sido relegada a un segundo plano. Sin embargo, los trabajos actuales de carácter más general sobre la obra plotiniana incluyen un tratamiento del tema que parece estar estableciendo una nueva tendencia en su interpretación respecto de las interpretaciones predominantes en las décadas anteriores. Por esta razón, ofreceremos, en tercer lugar, una presentación de las interpretaciones más recientes que los estudiosos han propuesto sobre el tema $y$, finalmente, pondremos de manifiesto las razones que nos llevan a disentir con la interpretación predominante del siglo pasado y a alinearnos con las más actuales.

\section{El discurso místico de Plotino.}

Antes de dedicarnos a examinar aquellos textos en los que Plotino presenta sus experiencias trascendentes es necesario introducir una aclaración sobre la noción de "mística". Este término no es propio de la filosofía plotiniana ni las palabras relacionadas poseen en las Enéadas el significado que, junto con numerosos estudiosos, le atribuimos para calificar la serie de experiencias trascendentes descritas por Plotino. En la Antigüedad, el término

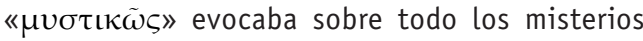
de Eleusis $^{2}$ y designaba los secretos que no eran accesibles sino a los iniciados. ${ }^{3}$ En las Enéadas, por su parte, encontramos una sola vez el adverbio $\mu v \sigma \tau \iota \kappa \tilde{\omega} \varsigma^{4}$ y una sola vez aparece también en la Vida de Plotino ${ }^{5}$ de Porfirio. A pesar de que el significado etimológico del término es "lo concerniente a los misterios", tanto Plotino como Porfirio lo utilizan para caracterizar un tipo de interpretación alegórica de mitos y de ritos. Esta interpretación tiene como modelo la práctica de los misterios y su finalidad es mostrar cómo los poetas que parecen hablar de la realidad sensible evocan, de hecho, la realidad inteligible que es objeto de la filosofía. ${ }^{6}$ A pesar de que la noción de "mística" tiene este significado en su origen, la tradición interpretativa lo ha aplicado a un aspecto de la filosofía de Plotino basándose en el significado que el término adoptó posteriormente con el cristianismo. Esta aplicación en su máxima expresión ha dado lugar a que se considere a Plotino el "padre de la mística occidental".
Ahora bien, es necesario caracterizar cómo comprendemos la noción de "mística" en este trabajo. En un sentido general, mística se define ${ }^{8}$ como la doctrina que afirma la posibilidad de una unión íntima y directa del espíritu humano con el principio fundamental de la realidad. Pero el término es aplicado también a la experiencia misma de una unión semejante y no sólo a la creencia de que es posible o a la doctrina que la sustenta. Esta unión constituye, simultáneamente, un modo de existir y un modo de conocer distintos y que son considerados superiores a la existencia y al conocimiento normales. ${ }^{9}$

En las Enéadas, por su parte, encontramos numerosos discursos que pueden ser calificados de "místicos" en ambos sentidos: tanto porque describen las experiencias trascendentes como porque ofrecen una doctrina que interpreta y explica esta clase de experiencias. Sin embargo, en estos discursos descripción e interpretación, narración y explicación se dan conjuntamente y son difícilmente discernibles, lo cual representa una primera dificultad para el estudio de aquellos. Otra dificultad propia de esta clase de discursos es advertida por Plotino quien, en numerosos pasajes, hace referencia a ella. Presentamos, a continuación, dos pasajes ilustrativos.

"En consecuencia, lo Uno es también verdaderamente inefable, puesto que digas de él lo que digas, estarás siempre diciendo algo. Sin embargo, [...] nada puede serle atribuido. Nosotros, empero, en tanto nos es posible, intentamos referirnos a él. ${ }^{10}$

"Así también nosotros ahora hablamos de lo Uno como si fuera algo diferente. Es por ello que la contemplación es dificilmente expresable ¿Cómo referirse a él como si se tratara de otra cosa, puesto que al contemplarlo no se lo estaba viendo como algo diferente sino como unidad consigo mismo?" ${ }^{11}$

Tanto el principio supremo de la realidad como la experiencia que se tiene en relación con ese principio son inefables y difícilmente expresables. Esto implica una dificultad insalvable en cuanto a la posibilidad de brindar una descripción adecuada y exacta de la experiencia. Al querer analizar los discursos místicos nos encontramos,
2. Tal significado lo encontramos, por ejemplo en Aristóteles: “... hay quien dice que una cosa se le escapó en la conversación, o que no sabía que era un secreto, como Esquilo con los misterios

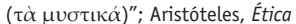
Nicomaquea III 1, 1111a.8-10

3. Hadot (1982).

4. III $6,19,26-29$

5. VP 15, 1-5

6. Brisson (2007: 453-459). En su artículo, el autor afirma que "el término mística... no puede ser utilizado para calificar la unión del alma con el primer Principio, designado como lo Uno o como el Bien".

\section{Rist (1967:213).}

8. Encontramos otra definición en Merlan (1963: 1) “Por definición consideramos que significa una doctrina que enseña que los momentos más elevados de la existencia del hombre son aquellos en los cuales se absorbe en aquello que él asume como divino... es una experiencia sui generis, que difiere de la experiencia humana común"

9. Cf. Dodds (1968: 100). Para un análisis detallado de los diferentes grados de conocimiento en

Plotino véase Santa Cruz (2006: 201-216).

10. V $3,13,1-6$

11. VI $9,10,19 \mathrm{ss}$. 
en consecuencia, con dos dificultades de suma importancia: en primer lugar, con la imposibilidad de discernir entre descripción e interpretación, es decir, entre experiencia y doctrina; en segundo lugar, con la imposibilidad de brindar un discurso ajustado y exacto de la experiencia mística y de lo Uno mismo por el carácter inefable de ambos. Frente a estas dificultades, cabe preguntarse por la viabilidad de un trabajo como el presente cuyo objetivo es el estudio y análisis de los discursos místicos expuestos en las Enéadas. Ahora bien, debe tenerse en cuenta que este interrogante surge, justamente, del estudio mismo de los discursos místicos plotinianos, por lo cual reconocer sus dificultades inherentes no impide comprenderlos sino que constituye un primer paso para lograrlo.

Por otra parte, la descripción de la experiencia y su interpretación presentes en los discursos místicos de las Enéadas constituyen una suerte de círculo hermenéutico en el que una es fundamento y, a la vez, explicación de la otra. Es decir, la doctrina metafísica está implicada en la descripción que Plotino hace de la experiencia pero, a su vez, la experiencia sirve de corolario y fundamento "empírico" último de la doctrina metafísica. ${ }^{12} \mathrm{Si}$ tenemos esto en cuenta, podemos afirmar que las dos dificultades que señalamos anteriormente no son meramente discursivas sino que tienen su raíz en la realidad misma que buscan expresar. Es decir, la dificultad para discernir entre doctrina y experiencia no radica en una limitación de Plotino para comunicar sus ideas ni en el lenguaje mismo sino en la concepción plotiniana de la realidad según la cual a cada grado de lo real postulado en su doctrina le corresponde una actividad noética y, por lo tanto, una experiencia diferente de la de los otros grados. A raíz de lo expuesto podríamos entender que la doctrina que propone diversos grados de lo real, cada uno de los cuales ejerce una actividad contemplativa peculiar, y la experiencia que el hombre puede obtener de estos grados participando de la actividad propia de aquellos son aspectos complementarios e inseparables en la filosofía de Plotino.

Finalmente, creemos que si bien Plotino es conciente, en alguna medida, de estas dificultades, no duda del valor psicagógico que poseen los discursos místicos y de la acción persuasiva que pueden tener sobre el alma de quien los recibe. En efecto, una de las finalidades del discurso místico plotiniano es invitar a los hombres a que se dispongan mediante la virtud a experimentar por ellos mismos los grados de la realidad que concibe nuestro filósofo. ${ }^{13}$

En la próxima sección nos ocuparemos de los discursos místicos plotinianos. No pretendemos aquí ofrecer un examen de todos los pasajes en los que Plotino expresa su mística sino que nos limitaremos al análisis de un número acotado de citas textuales pero que, a nuestro entender, son suficientemente explícitas y relevantes. En este trabajo partiremos de dos hipótesis. La primera hipótesis sostiene que es posible interpretar los discursos plotinianos sobre la experiencia trascendente de modo de extraer de ellos una doctrina mística coherente. Nuestra segunda hipótesis, por cierto mucho más comprometida, es que Plotino mismo brinda en sus discursos su propia doctrina mística. Por consiguiente, nuestro propósito es, a la vez, ambicioso y modesto. Ambicioso, porque pretende poner de manifiesto la doctrina mística plotiniana. Modesto, porque de no lograr nuestro propósito más ambicioso, las conclusiones de este trabajo ofrecerán una interpretación posible y fundamentada de los discursos místicos plotinianos.

\section{Diversidad de experiencias, diversidad de discursos.}

Si consideramos que para Plotino la realidad está constituida por diferentes niveles llamados hipóstasis a los cuales el hombre puede acceder modificando su actividad noética, debemos afirmar que todo contacto con las realidades superiores al Alma, nivel en que el hombre desarrolla su actividad dianoética usual, constituye una experiencia mística. Por esta razón, algunos estudiosos han llamado al contacto con la Inteligencia misticismo noético ${ }^{14}$ para distinguirlo de la experiencia de unidad. ${ }^{15}$ Plotino, en efecto, afirma:

"Es menester, por lo tanto, hacerse inteligencia y confiar y someter la propia alma a la inteligencia para que aquella, estando despierta, reciba lo que ésta ve..$^{16}$ 
En este pasaje, Plotino explica la posibilidad del hombre de identificarse con su inteligencia y participar de su actividad, pero esta actividad no se identifica con la del alma sino que posee un grado mayor de unificación con su objeto y es no discursiva. Es a raíz de esto que los estudiosos lo han considerado como un primer estadio místico. A continuación transcribiremos otro pasaje del mismo tratado en el cual Plotino afirma qué clase de relación es posible con lo Uno y que su característica propia, a diferencia de la unión carnal, es que no hay un mero contacto externo.

“El que no tenga experiencia de ello, colija de acá y de los amores de acá cuál será en encuentro con el amor de sus amores; sepa que los amados de acá [...] no eran el verdadero amado, ni el Bien nuestro que buscamos. Allá en cambio, está el verdadero Amado, con el que podemos incluso unirnos participando de él y poseyéndolo realmente y no abrazándolo por fuera carnalmente. ${ }^{17}$

Así como en el pasaje anterior Plotino habla de que el alma se "vuelva inteligencia" y "reciba lo que esta ve", en este pasaje hace hincapié en la unión con lo Uno. Es posible pensar, debido a esto, que se alude a experiencias diferentes. Ahora bien, para analizar las diferentes experiencias místicas es necesario tener en cuenta la concepción plotiniana de alma humana. Para Plotino el alma del hombre es, por una parte, una entidad propia de un determinado nivel de la realidad pero, por otra, es la potencia capaz de recorrer de un extremo a otro la cadena de realidades y de asimilarse a cada una de ellas mediante una serie de transformaciones ${ }^{18}$ en su actividad noética. Esta segunda concepción del alma es la que permite a Plotino afirmar que el hombre puede elevarse desde la realidad sensible hacia las superiores transformando sus estados de conciencia. El alma, entendida de este segundo modo, puede acceder a la Inteligencia en primer lugar y a lo Uno en segundo lugar. En el presente trabajo nos limitaremos al estudio de la experiencia mística última, es decir, aquella que involucra a lo Uno en sí mismo. Ahora bien, Plotino brinda varias descripciones con relación a las "experiencias místicas" que involucran a lo Uno las cuales es preciso distinguir.

Nuestro filósofo afirma que la Inteligencia posee dos potencias mediante las cuales puede contemplar, tanto a las Ideas como a lo Uno, ${ }^{19} \mathrm{y}$ que el alma, una vez que accede a aquella, puede contemplar lo que la inteligencia ve. La primera de estas potencias no ofrece demasiadas dificultades ya que el alma contempla las Ideas instalándose en la Inteligencia. Mediante esta actividad el hombre logra contemplar su yo ideal retornando a la fuente de su identidad, si no personal, al menos humana. ${ }^{20}$ La segunda potencia, la que pretende establecer una relación entre la Inteligencia y lo Uno, presenta, por el contrario, numerosos problemas. Plotino, asimismo, relaciona esta segunda potencia con la actividad contemplativa, por lo cual surge el problema de si lo Uno es efectivamente contemplable por la Inteligencia, posibilidad que Plotino parece, por momentos, negar. A modo de ejemplo, citemos los siguientes pasajes:

1) "Cuando digo que [el pensamiento] no podría ser el pensamiento del Bien, no quiero decir que no sea posible pensar al Bien-admitamos, en efecto, que ello es posible-sino que no puede haber pensamiento en el Bien mismo." VI 7, 40, 33-35.

2) "La inteligencia, para ser inteligencia, debe mirar hacia lo Uno. Pero lo ve, no porque esté separada de él, sino porque viene después de lo Uno, sin que haya intermediarios." V 1, 6, 47-48.

3) "Es así que esta Inteligencia múltiple, cuando intenta pensar lo que está más allá, quiere pensar a esto mismo más allá como una unidad; pero aunque quiere apresarlo en su simplicidad, acaba siempre por recibir en sí misma algo diferente, que se ha pluralizado." V 3, 11, 1-3.

En los primeros dos pasajes se hace referencia, claramente, a la intuición intelectual de lo Uno por parte de la Inteligencia, descrita en el primero como "pensar" $y$, en el segundo, como "mirar" y "ver". En el tercer pasaje, Plotino afirma que no es posible obtener una intuición intelectual de lo Uno tal como lo Uno es, en su simplicidad absoluta, sino que en el intento de obtener una intuición semejante se
17. VI $9,9,43-45$

18. Bréhier (1926: 74).

19. Por ejemplo, en VI 9, 2, 34-45 y 3, 32-37

20. Aun cuando no desconocemos su importancia, no es nuestra intención abordar el problema de la existencia de Formas de individuos en Plotino. Para una discusión detallada sobre este punto véase Blumenthal (1973:112-133) y Kalligas (1997: 206-223). Nos limitamos a afirmar aquí que en la Inteligencia el hombre encuentra al menos su Forma de Hombre mediante la cual conserva su identidad como tal. 
pluraliza a lo Uno y se obtiene un objeto de visión y de pensamiento múltiple. Es evidente que Plotino traza una distinción entre el objeto de la intelección en sí mismo y la representación que el sujeto que

21. Santa Cruz (2007: XLVII). 22. $V 4,2,7$. 23. V $3,11,10,13$. 24. V 3, 11, 10-11. 25. $\vee 4,2,6$. 26. $\vee 9,8,1$.

27. V 3, 11, 14-15. Tal vez pueda identificarse con la Díada indeterminada o con la materia inteligible (II 4, 4-5 y V 1, 5). De todos modos, este pasaje no debe entenderse con un sentido temporal sino, más bien, como la distinción entre estados noéticos progresivamente simplificados.

28. VI $7,35,20$.

29. V 3, 10, 40ss.

30. Un análisis detallado de la metáfora del contacto merece un trabajo completo pero no es ese el objetivo del presente artículo.

Hemos introducido aquí este pasaje para mostrar que Plotino alinea esta serie de metáforas con la experiencia que la Inteligencia en su estado indeterminado puede tener de lo Uno.

31. VI 9, 11, 6-7. Santa CruzCrespo (2007: 272-273) traducen "como ya no eran dos, sino que quien veía era él mismo, una unidad con el objeto de su visión".

Cabe preguntarse si Plotino se refiere a que en este estado se es uno con la imagen que se ve o si es uno con el "objeto" al que se había tendido la mirada. Tal vez la aclaración que sigue define la cuestión: "sería mejor hablar no de objeto de visión sino más bien de objeto de unión". intelige tiene de ese objeto. ${ }^{21}$ En el caso de la Inteligencia, esta quiere captar a lo Uno tal como él es pero la representación que posee de aquel es diferente de lo Uno mismo.

En relación con la distinción recién trazada, Plotino afirma que en la Inteligencia hay dos clases de visión diferentes. En primer lugar, una "visión sin impronta" 0 "intelección indefinida" ${ }^{22}$ $\mathrm{y}$, en segundo lugar, una "visión con impronta", "visión que ve" ${ }^{24}$ "intelección definida". ${ }^{25} \mathrm{La}$ visión que pluraliza descrita en el pasaje 3) es propia de la intelección definida y el resultado de esta intelección es la contemplación de las Formas, objeto de pensamiento propio de la Inteligencia ${ }^{26}$ que es, a su vez, contemplación de sí misma. En el caso de la visión sin impronta, no es exacto afirmar que el sujeto que desarrolla esta actividad es la Inteligencia, puesto que se vuelve Inteligencia recién cuando logra apresar al objeto y comienza a pensarlo. ${ }^{27}$ Sin embargo, Plotino habla, asimismo, de dos potencias de la Inteligencia. ${ }^{28}$ La primera es

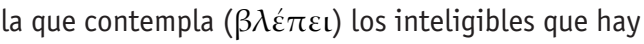
dentro de ella misma y la otra es la que contempla al que está más allá mediante una aprehensión y

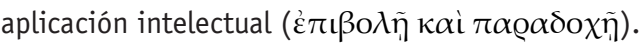

Atendiendo a lo que acabamos de señalar podemos asociar todas estas nociones: Inteligencia (propiamente dicha), visión con impronta, contemplación de la imagen de lo Uno en la Inteligencia, contemplación de las Formas, contemplación de sí mismo y primer potencia de la Inteligencia. Y, por otra parte, estas otras nociones: segunda potencia de la Inteligencia (entendiendo Inteligencia de un modo laxo) y mirada sin impronta. Respecto de las dos clases de visión Plotino también afirma lo siguiente:

"Es preciso que lo pensante aprehenda algo diverso y que lo pensado, por ser objeto de aprehensión del pensamiento, sea variado. De lo contrario, no habría intelección de sí mismo, sino un tacto $y$, por así decirlo, un mero contacto inefable y no pensante: una pre-intelección, pues quien toca no piensa, todavía no devino inteligencia." 29

El pensamiento de sí de la Inteligencia precisa de un objeto mientras que, cuando se halla privada de su objeto, no es posible afirmar que hay pensamiento sino "pre-pensamiento", actividad característica del estadio de indeterminación de la Inteligencia que se describe en este pasaje mediante la metáfora del contacto. Con esta metáfora Plotino alude, entonces, a la aprehensión pre-intelectiva que la visión indeterminada de la "Inteligencia" puede obtener de lo Uno.

Ahora bien, es preciso tener en cuenta que con estas descripciones Plotino se refiere a la Inteligencia hipóstasis. Sin embargo, muchos estudiosos las han asociado, con razón, a la experiencia mística del hombre. Consideramos que es posible afirmar que esta descripción también es válida para el hombre si se tiene en cuenta que la inteligencia del hombre toma parte de la actividad de la Inteligencia hipóstasis y que el hombre puede identificarse con su inteligencia para "ver lo que aquella (la Inteligencia) ve".

Junto con estas caracterizaciones de la experiencia mística, Plotino brinda otra serie de discursos que nos llevan a entender la experiencia trascendente última de un modo diferente. En el tratado VI 9 afirma:

"Puesto que no eran dos cosas, sino que el vidente mismo era una sola cosa con lo visto -diríase no visto,

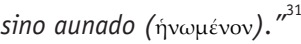

En este pasaje encontramos no solamente un ejemplo de cómo Plotino reconsidera su caracterización de la experiencia en términos de "visión" sino además que se establecen dos nociones diferentes: por un lado, el vidente $y$ lo visto se han aunado $\mathrm{y}$, por otro, son una sola cosa. Estas dos nociones descartan la posibilidad de que persista algún grado de dualidad entre el místico y lo Uno. No se hace referencia aquí a la inteligencia que se contempla a sí misma ni a la inteligencia indeterminada que "contacta" a lo Uno, sino a un grado de unificación completa en el cual 
hay aunamiento, volverse uno: !íùiYiiiiir. En este momento de la experiencia, el místico, en cuanto a su actividad "hiperintelectiva", se ha transformado en lo Uno.

"Como transformado en otro y no siendo él mismo de sí mismo, <el místico> es anexionado a aquél < a lo Uno>, y hecho pertenencia de aquél, es una sola cosa con él. ${ }^{132}$

Mediante esta nueva serie de discursos Plotino supera ambas caracterizaciones que describen la relación mística del hombre con lo Uno en términos de visión y de contacto. La visión, por una parte, implica una distancia entre el objeto y el sujeto y el contacto, por otra parte, implica una cercanía y un roce, por así decirlo, de las superficies. La unión, por su parte, anula la distancia y trasciende el rozamiento para devenir en una conjunción de centros en la cual los dos centros son ya uno. Mediante esta nueva caracterización, Plotino supera la dualidad presente en los discursos tratados anteriormente e introduce la posibilidad de que el hombre experimente, tal como le es posible, lo Uno.

Ahora bien, ¿cómo es posible para el hombre “experimentar" lo Uno? En el caso de la Inteligencia y de su actividad auto-contemplativa, el hombre podía participar de esta actividad gracias a su propia inteligencia. Pero, ¿debe limitarse la “experiencia de lo Uno" humana a la que obtiene su propia inteligencia o hay en el hombre una potencia superior mediante la que puede acceder a lo Uno? Plotino evidencia haber pensado estas cuestiones, respecto de las que afirma:

"Ahora bien, del mismo modo que esta Trinidad de la que hemos hablado existe en la naturaleza, asi hay que pensar que también habita en el hombre.".

Así como el Alma, la Inteligencia y lo Uno son los grados constitutivos de la realidad son, asimismo, los grados constitutivos de la estructura psíquica del hombre.

"Es preciso que exista en nosotros una inteligencia [...] y que también exista el Principio y la Causa de la inteligencia, o sea Dios, sin que ese Dios esté dividido." ${ }^{35}$

Considerando lo expuesto en los últimos dos pasajes citados, podríamos entender que Plotino afirma que lo Uno está presente en el hombre y que le es inmanente. En el primer pasaje se sostiene la presencia de las tres hipóstasis en el hombre $y$, en el segundo, la presencia de lo Uno porque, según creemos, el principio y causa de la inteligencia al que Plotino llama allí Dios, es lo Uno. Ahora bien, la presencia de lo Uno en el hombre es tal que lo Uno hipóstasis y lo Uno en el hombre no son algo diferente, pues de no ser así lo Uno estaría dividido. Por el contrario, Plotino parece sostener que la modalidad de la presencia de lo Uno en cada hombre es tal que lo Uno hipóstasis no se divide ni fragmenta por estar presente en un hombre en particular, ni por estar presente en la multiplicidad de hombres. Lo Uno en el hombre y lo Uno hipóstasis son, pues, Uno.

“¿Con qué intuición instantánea podríamos captar a aquel, puesto que sobrepasa la naturaleza de la inteligencia?...le responderemos que con lo que hay en

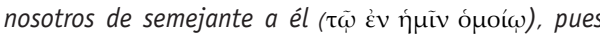

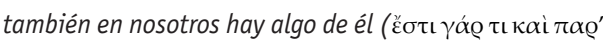

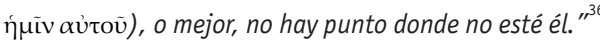

En este pasaje Plotino indica que obtendremos la experiencia suprema mediante la potencia presente en nosotros que se asemeja a lo Uno. El uso del término "semejante" podría dar lugar a la suposición de que se refiere a la Inteligencia indeterminada, sin embargo, las dos aclaraciones que Plotino agrega a continuación, "algo de él" y luego "no hay punto donde no esté él", nos hacen descartar esa suposición. Plotino se refiere aquí, a nuestro entender, a lo "Uno en el hombre", aquello de él presente en nosotros que, a su vez, es lo Uno mismo. De este modo, la presencia de lo Uno en el hombre permite a Plotino explicar la experiencia mística suprema e interpretarla, a su vez, como una experiencia de unión o unidad absoluta puesto que el místico accede a la experiencia del ámbito de su interioridad donde lo Uno está presente.
33. La metáfora de los centros también es utilizada por Plotino para expresar la idea de unión final. Citemos, a modo de ejemplo, el siguiente pasaje: “Así nosotros, descollando con aquello de nosotros que no está sumergido en el cuerpo, con eso coincidimos por nuestro propio centro con el cuasi-dentro de todas las cosas, hallando así reposo." VI 9, 8 , 19-20.

34. V 1, 10, 1-7.

35. V 1, 11, 5-9.

36. III 8,9 , 20 ss. 
37. Armstrong (1940: cap. III).

38. VI 7, 35, 24-27

39. Entre los especialistas que aceptan esta caracterización como referida a la experiencia mística última se encuentran Bréhier (1926:184), Rist (1967: 228), Hadot (1982-3: 462), Armstrong (1976: 96), Igal (1982: 99), Santa Cruz (2007: LXXX-LXXXIII), Brisson(2007: 464), entre otros. Algunos estudiosos que no aceptan esta caracterización, la interpretan de otro modo o no la identifican con la experiencia mística última son, por ejemplo, 0’Daly (1973: 88 y 1974: 2-3), Meijer (1992: 317-318) y Gerson (1994: 223, 293 n. 50,51) entre

otros.

40. Aun así, autores como Armstrong (1940: 29-47) e Igal (1982: 99-100), quienes aceptan la caracterización de la unión mística como un estado de la inteligencia enamorada, sostienen que es posible encontrar, asimismo, otros pasajes que describen una unión que conlleva verdadera identificación.

41. Armstrong (1940: 31) afirma que cuando lo Uno es tomado como Dios y como el objeto trascendente de una experiencia religiosa, no es posible pensar a la unión mística como la realización de una identidad preexistente, identidad afirmada por Plotino frecuentemente, según el autor.

42. Zaehner (1957), citado en Rist (1967: 213-214).

43. Podríamos agregar nosotros que el Intelecto por definición sería incapaz de abandonar las formas, pero esto no implica que

el hombre sea, por definición, incapaz de dejar de ser intelecto y, por lo tanto, de dejar ir las formas.

44. Hadot (1963: 100-101).

45. Del mismo modo interpreta Armstrong (1976: 196) estos elementos de la filosofía plotiniana cuando dice que la unión del alma del místico es una perfecta asimilación a la unión eterna que el Intelecto hipóstasis disfruta con lo Uno en un estado de locura, amor y ebriedad.

\section{Diversidad de interpretaciones}

Ahora bien, así como Plotino presenta una diversidad de experiencias trascendentes (relacionadas con las dos actividades de la inteligencia o con lo Uno) y ofrece, asimismo, diferentes discursos sobre estas experiencias, del mismo modo, los estudiosos han propuesto diversas interpretaciones de la experiencia mística. En efecto, no todos los intérpretes coinciden en que Plotino distingue varios niveles de experiencias místicas. Algunas interpretaciones, por ejemplo, consideran que uno de los niveles descritos representa la única clase de experiencia mística y que las diversas descripciones restantes deben considerarse contradictorias e inconsistentes con aquella otra. ${ }^{37}$

Como pusimos de manifiesto en la sección anterior, Plotino afirma que la potencia superior de la Inteligencia se vuelve sobre lo Uno para contemplarlo mediante una visión indeterminada. Esta caracterización de la experiencia mística ha sido objeto de numerosas interpretaciones y suele asociarse con el siguiente pasaje:

"La primera es la contemplación propia de una inteligencia cuerda; la segunda es inteligencia

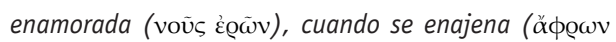
$\gamma \varepsilon ́ v \eta \tau \alpha \iota)$ embriagada de néctar; y entonces es cuando, desencogida y eufórica por la saturación se vuelve inteligencia enamorada. ${ }^{\prime 38}$

Aquí Plotino identifica la potencia superior de la Inteligencia con una Inteligencia enamorada, embriagada y eufórica. Algunos estudiosos han entendido que Plotino realmente caracteriza de este modo la experiencia mística última. ${ }^{39}$ Es de notar que estos intérpretes no postularían, aparentemente, una completa identificación del místico con lo Uno ya que entre Inteligencia y Uno siempre habrá algo que los diferencie. ${ }^{40}$ Esta interpretación puede ser caracterizada como teísta, ${ }^{41}$ si entendemos por este término, siguiendo a Zaehner, una experiencia en la cual se realiza una unión del alma con el Dios trascendente pero sin que el alma sea, ni antes ni durante la unión, idéntica a Dios. ${ }^{42}$ Asimismo, al ser una experiencia de amor que requiere de un amante y un amado, siendo lo Uno - Dios el amado y la inteligencia el amante, la dualidad también está implicada en este momento último de la experiencia.

0 'Daly, por el contrario, argumenta que

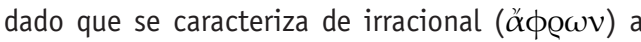
la inteligencia amante, no puede aceptarse que se trate de una inteligencia y que el mismo Plotino encuentra esta noción insatisfactoria. Para explicar la experiencia mística recurrirá a otra solución basándose en las descripciones plotinianas del estado indeterminado de la Inteligencia en el primer estadio de su generación. 0`Daly encuentra que lo que obtiene la visión de lo Uno es aquella potencia que en un estadio posterior se convertirá en intelectiva. Considera, a su vez, que el momento primordial en que la Inteligencia se vuelve hacia lo Uno y lo capta de modo pre-intelectivo antes de generar las Formas es descrito con el mismo vocabulario que se utiliza para describir la visión mística. Sostiene, a modo de conclusión, que este uso paralelo del vocabulario permite establecer una analogía entre ambas descripciones explicando la segunda en los términos de la primera. Otra interpretación provee Gerson, quien reconoce que Plotino afirma que se conoce cómo es lo Uno cuando la inteligencia abandona las Formas, es decir, tras renunciar a todo lo inteligible; sin embargo, no concuerda con que esto pueda interpretarse en sentido literal pues un Intelecto eterno, sin comienzo temporal alguno, es verdaderamente incapaz de renunciar a las Formas. ${ }^{43}$ Hadot, $^{44}$ por otra parte, afirma que el proceso cosmogónico a través del cual el noûs se vuelve hacia el Bien y engendra las Formas y los seres se sitúa en nosotros mismos. Estos niveles de la realidad, sostiene, son al mismo tiempo los niveles de nuestro yo, por lo cual la experiencia mística se realizará cuando el alma tenga la posibilidad de vivir con el noûs el éxtasis amoroso que lo hace nacer, entrando en un gozoso contacto con el Bien. ${ }^{45}$

Todas estas interpretaciones limitan al místico, en su experiencia última, al nivel de la Inteligencia. Aunque puede variar la interpretación al considerarse que la Inteligencia es incoada o ebria, no se logra superar el nivel de la segunda hipóstasis. Por otra parte, estas opciones tampoco 
dan cuenta de las afirmaciones de Plotino referentes a la unión total con lo Uno, en la cual el alma es una sola cosa con aquél. Quienes aceptan aquellas interpretaciones suelen quitar valor a las descripciones de unión alegando que presentan una doctrina contradictoria con la teísta predominante, ${ }^{46}$ que ocupan un espacio relativamente pequeño en las Enéadas ${ }^{47}$ o que no se expresan con un "lenguaje extremo" propio de las doctrinas no dualistas o monistas. $^{48}$

En relación con esta identificación entre la experiencia mística última y una de las fases de la generación de la Inteligencia, Meijer ${ }^{49}$ niega que puedan ser identificadas. Afirma, en efecto, que si

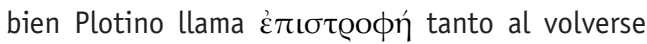
de la mente universal hacia lo Uno, movimiento que conlleva la generación de las Ideas, como a la vuelta del alma a su principio, no debe creerse que la primera tenga alguna participación o rol en el retorno del alma particular. Fundamenta esto argumentando que la contemplación productiva que va a ejercer el Intelecto todavía incoado cuando se vuelve hacia lo Uno no tiene como finalidad la unión $^{50}$ sino que es un volverse contemplativo.

Ahora bien, no han faltado intérpretes que han reparado en los discursos místicos que se refieren a la experiencia trascendente en términos de unión atribuyéndoles un lugar relevante en la doctrina de Plotino. Estas interpretaciones han sido muy discutidas durante el siglo pasado pero en los últimos años han encontrado numerosos adherentes.

Ya en 1940 Armstrong había notado que Plotino proponía tres formas de teología trascendente, cada una con su concepción de lo Supremo bastante diferente, una de las cuales implicaba la identidad absoluta entre el yo del místico y lo Uno en el momento máximo de la experiencia mística. ${ }^{51}$ Armstrong, sin embargo, consideró que con esta presentación triple Plotino incurría en una inconsistencia y en una contradicción. Recién en 1976 Mamo defendió en un artículo la interpretación monista de la mística eneádica sosteniendo que lo Uno plotiniano es inmanente y que, por lo tanto, en la experiencia mística el hombre se retrotrae a su verdadero origen con el cual se identifica. Aun así, reconoce que lo Uno es, a su vez, trascendente $y$, por lo tanto, no puede considerarse a Plotino como un monista "rígido". ${ }^{52}$ Otra autora que sostuvo una interpretación no dualista es Gatti y, en relación con esto, recuerda la afirmación de Plotino según la cual la comunión suprema no es con una estatua, ni con una imagen, sino como lo Uno mismo. ${ }^{53}$ Finalmente, recién en los últimos años la interpretación monista ha encontrado una creciente adherencia entre los intérpretes. Algunos de los estudiosos que en sus trabajos más recientes aceptan la completa identificación que el hombre plotiniano experimenta con lo Uno en el éxtasis místico son Carone, ${ }^{54}$ Ousager, ${ }^{55}$ Reemes $^{56}$ y Chiaradonna. ${ }^{57}$ En relación con la experiencia mística última Reemes escribe:

"El telos último, la unificación con lo Uno, involucra una pérdida del sí mismo. Lo que se requiere es un abandono de todos los límites, incluyendo todos los caracteres personales, así como rendirse a un poder más vasto que uno mismo. La transformación del sí mismo en bondad absoluta, unidad y poder infinito es acompañada por la pérdida de su particularidad y auto-determinación".

En este breve texto, la autora pone de relieve una interpretación de la experiencia mística semejante a la que hemos presentado en la primera parte de nuestro trabajo. Por otra parte, las consecuencias que esta interpretación tiene sobre la identidad del hombre son muy diferentes a la de los trabajos de los autores antes citados que defienden una interpretación teísta o dualista. Las lecturas que reconocen en Plotino una superación del dualismo en la experiencia mística final logran dar cuenta de las exposiciones plotinianas más comprometidas con la unidad y proporcionan, asimismo, una caracterización de la doctrina mística plotiniana más coherente que aquellas que descartan o desvalorizan una parte de las exposiciones de Plotino. La tendencia interpretativa actual parece estar acercándose al texto con menos preconceptos sobre cómo debe ser y entenderse la mística del filósofo que inspiró a los místicos cristianos y le otorga una mayor libertad que consiste en no silenciar algunos pasajes e integrarlos en la comprensión de nuestro filósofo y de la doctrina mística que ofrece. ${ }^{59}$
46. Armstrong (1940: 43).

47. Armstrong (1940: 44).

48. Rist (1967: 226).

49. Meijer (1992: 310, nota 878).

50. Meijer (1992: 305 y n. 864).

51. Armstrong (1940: capítulo III). Si bien afirma preferir la concepción "positiva" de lo Uno, como central de la filosofía de Plotino.

52. Mamo (1976: 199: 215).

53. Gatti (1982: 164).

54. Carone (1997: 177-187).

55. Ousager (2005: cap. I.C.).

56. Reemes (2007: cap.6), (2008: cap. 4).

57. Chiaradonna (2009: 172-175).

58. Reemes (2008: 133).

59. Ya Mamo (1976: 200-1) había puesto en evidencia los juicios valorativos que guiaban el análisis de la mística plotiniana de algunos interpretes debido a la presencia de ciertos elementos místicos que contradecían a teología cristiana. 


\section{Conclusiones}

En el presente artículo hemos presentado y defendido una interpretación posible de los diferentes discursos místicos que Plotino expone en sus Enéadas. Hemos puesto de relieve los problemas que estos discursos presentan e intentamos explicar la causa de estas dificultades. Hemos procurado demostrar, asimismo, que la diversidad de discursos corresponde a una diversidad de experiencias y que es posible sostener simultáneamente y sin incurrir en una inconsistencia diferentes descripciones de la experiencia mística por tratarse de diferentes niveles de esta clase de experiencias. En tal sentido, hemos creído importante señalar que los discursos en los que se emplea el vocabulario de la visión y del contacto pueden ser considerados

60. Nos referimos aquí a las caracterizaciones en términos de "visión", "contacto" y “unión". como muestra de una doctrina coherente y que no son contradictorios entre sí ni con el sistema metafísico de Plotino. Por otra parte, argumentamos a favor de la interpretación que entiende que los discursos en los que predomina el vocabulario de la unión describen la experiencia mística final en la cual el místico se unifica con lo Uno gracias a que logra acceder, mediante la modificación de sus estados noéticos, a la presencia de lo Uno en sí mismo. Esta caracterización de la experiencia mística tampoco entra en contradicción con las otras caracterizaciones por tratarse de una actividad psicológica con un nivel de unidad absoluto, ausente en las otras actividades. Concluimos, en consecuencia, que toda la serie de discursos tratados son muestra de la doctrina mística plotiniana y que esta es de carácter coherente, en tanto describe detalladamente los diferentes estados noéticos por los que atraviesa el hombre en su simplificación hacia la unión final. En efecto, la doctrina mística de Plotino sostiene que en un primer momento el hombre se identifica con su inteligencia gracias a lo cual logra contemplar las Formas. En un segundo momento, el hombre actualiza una potencia diferente de su Inteligencia mediante la cual logra un contacto inefable con lo Uno. En tercer lugar, el hombre se identifica con lo Uno presente en sí mismo al simplificar de modo absoluto su actividad noética gracias a lo cual experimenta el estado de unidad que Plotino describe en términos de unión con lo Uno.

Finalmente, nuestro examen de las interpretaciones de la mística plotiniana predominantes tanto en el siglo pasado como en los últimos años nos ha permitido tanto contextualizar nuestro análisis con los trabajos de los especialistas como poner en perspectiva la actividad de los intérpretes mismos. Esta perspectiva nos permitió, asimismo, advertir las tendencias interpretativas y el progreso que evidencian las interpretaciones más recientes respecto de las anteriores, progreso que se manifiesta en una justa apreciación de la diversidad de los discursos de Plotino sobre las experiencias místicas sin que una caracterización sea preferida en detrimento de la otra. En nuestra interpretación, que creemos pone de manifiesto la doctrina mística plotiniana, todas las caracterizaciones ${ }^{60}$ presentes en las Enéadas son consideradas y analizadas por lo cual a cada una se le adjudica una función y un orden dentro del sistema plotiniano.

La interpretación de la filosofía de Plotino sigue siendo un proceso abierto y en desarrollo y esperamos haber contribuido, en alguna medida, a esclarecer algún punto de su doctrina y de la tarea exegética que a ella le dedica sus esfuerzos.

\section{Bibliografía citada}

ARAUJ0, M. y MARÍAS, J. (1981), Aristóteles, Ética A Nicómaco, Traducción Introducción y Notas de -, Madrid, Centro de Estudios Constitucionales.

ARMSTRONG, A. H. (1976), "The Apprehension of Divinity in Plotinus", en Baine Harris, R. (ed.), The Significance of Neoplatonism, Norfolk, Old Dominion University, pp $187-198$

ARMSTRONG, A. H. (1940), The Architecture of the Intelligible Universe in the Philosophy of Plotinus: An Analytical and Historical Study, Cambridge, Cambridge University Press.

BEIERWALTES, W. (1995) Autoconoscenza ed esperienza dellUnità: Plotino, Enneade V 3. Roma, Vita e Pensiero.

BLUmenthal, H. J. (1973), Plotinus Psychology, His doctrines of the embodied soul, The Hague, Martinus Nijhoff.

BRÉHIER, E. (1926), La Filosofía de Plotino, Buenos Aires, Editorial Sudamericana.

BRISSON, L. (2007), “Pode-se Falar de União Mística em Plotino?", en Kriterion, nro. 116, pp. 453-466.

CARABINE, D. (1997), “The Mystical Journeys of Plotinus 
and Gregory of Niza", en Cleary, J. (ed.), The Perennial Tradition of Neoplatonism, Leuven, Leuven University Press, pp 188 - 199.

CARONE, G. R. (1997), “Mysticism and Individuality: a Plotinian Paradox", en Cleary, J. (ed.), The Perennial Tradition of Neoplatonism, Leuven, Leuven University Press, pp 177 - 187.

ChIARAdONNA R. (2009), Plotino. Pensatori, Roma, Carocci editore,

DODDS, E. R. (1968), Paganos y Cristianos en una Época de Angustia, Madrid, Ediciones Cristiandad.

GATTI, M. L. (1982), Plotino e la Metafisica della Contemplazione, Milano, CUSL.

GERSON, L. P. (1994), Plotinus, London, Routledge.

HADOT, P. (1980), “Les Niveaux de Conscience dans les États Mysitiques selon Plotin", en Journal de Psychologie, nro. 2-3, pp $243-266$.

HAD0T, P. (1963), Plotin u la Simplicité du regard, Paris, Gallimard.

HADOT, P. (1982-1983), “Histoire de la Pensée Hellénistique et Romaine", Annuaire du Collège de France, pp 459 - 465.

IGAL, J. (1982 - 2006), Plotino, Eneadas. Introducciones, traducción y notas de -, Madrid, Gredos, 3 vols.

KALLIGAS, P. (1997), "Forms of Individuals in Plotinus: A Re - Examination", en Phronesis, vol. XLII, nro 2, pp 206-223.
MAM0, P. (1976), “Is Plotinian Mysticism Monistic?", en Baine Harris, R. (ed.), The Significance of Neoplatonism, Norfolk, Old Dominion University, pp $199-215$.

MEIJER, P. A. (1992), Plotinus on the Good or the One, An Analitical Comentary, Amsterdam, Gieben.

MERLAN, P. (1963) Monopsychism, Mysticism, Metaconsciousness. La Haya, Martinus Nijhoff,

0'DALY, G. J. P. (1973), Plotinus Philosophy of the Self, Ireland, Harper \& Row Publishers.

O'DALY, G. J. P. (1974), "The Presence of the One in Plotinus" en Plotino e il neoplatonismo, pp. 159-169

OUSAGER, A. (2005), Plotinus, On Selfhood, Freedom and Politics, Denmark, Aarhus University Press.

RIST, J. M. (1967), Plotinus, The Road to Reality, Cambridge, CUP.

REMES, P. (2007) Plotinus on Self. The Philosophy of the 'We', Cambridge, Cambridge University Press.

REMES. P. (2008) Neoplatonism, Durham, Acumen.

SANTA CRUZ, M. I. (2006), "Modos de conocimiento en Plotino", en Estudios de Filosofía, n³4, pp. 201-216.

SANTA CRUZ, M. I. y CRESPO, M. I. (2007), Plotino, Eneadas, Textos Esenciales. Traducción, notas y estudio preliminar de - , Buenos Aires, Colihue. 